\title{
Multicenter, randomized, double-blind clinical trial to evaluate efficacy and safety of combined glucosamine sulfate and chondroitin sulfate capsules for treating knee osteoarthritis
}

Andrea Barranjard Vannucci Lomonte ${ }^{1 *}$ (D) José Alexandre Mendonça ${ }^{2}$, Gilberto de Castro Brandão ${ }^{3}$ and Marise Lazaretti Castro ${ }^{4}$

\begin{abstract}
Objectives: To compare the efficacy and safety of a new fixed dose combination of glucosamine sulfate and chondroitin sulfate capsules (GS/CS) versus the fixed dose combination of glucosamine hydrochloride and chondroitin sulfate (Cosamin DS ${ }^{\oplus}$ ) in capsules in patients with osteoarthritis $(\mathrm{OA})$ of the knee.

Methods: Multicenter, randomized, double-blind study. Participants with knee OA Kellgren-Lawrence grades 1 to 3 and VAS of symptoms $\geq 4 \mathrm{~cm}$ were randomized to receive GS/CS or Cosamin DS ${ }^{\circledR}$ over 12 weeks. The primary efficacy endpoint was the evaluation of the analgesic efficacy by the investigator. Secondary efficacy endpoints included: joint pain and swelling, investigator efficacy of the medication, and the use of rescue medication. Adverse events and drug tolerability were analyzed.
\end{abstract}

Results: One hundred patients were randomized, and 50 patients were allocated to each group. The analgesic efficacy evaluated by the investigator in the GS/CS group was 88.9, 95\%Cl: $75.2,95.8 \%$ and in the Cosamin DS ${ }^{\circledast}$ group was 85.4\%; $95 \% \mathrm{Cl}: 70.1,93.4 \%$. The mean reduction in the pain intensity was significant in both groups $(p<0.001)$, with no difference between them. The primary efficacy analysis demonstrated the non-inferiority of the GS/CS group compared with the Cosamin DS ${ }^{\circledast}$ group; the lower limit of the $90 \%$ confidence interval $(\mathrm{Cl})$ between the two groups $(-8.39 \%)$ was higher than the established margin of non-inferiority of $-10.00 \%$. Improvement in other efficacy outcomes was observed, again without differences between groups. Adverse events were similar between groups and both presented good tolerability.

Conclusions: The new fixed-dose formulation of GS/CS is effective in treating knee OA, presenting a good safety and tolerability profile.

Trial Registration: (https://clinicaltrials.gov/ct2/show/NCT00955552?term=NCT00955552\&rank=1; ClinicalTrials.gov; register number NCT00955552; First randomized patient: 08/17/2010).

Keywords: Glucosamine, Chondroitin, Osteoarthritis, Knee, Pain

\footnotetext{
* Correspondence: andrea.lomonte@cepic.com.br

${ }^{1}$ Centro Paulista de Investigação Clínica (CEPIC), Rua Moreira e Costa, 342 -

Ipiranga, São Paulo, SP 04266-010, Brazil

Full list of author information is available at the end of the article
}

(c) The Author(s). 2018 Open Access This article is distributed under the terms of the Creative Commons Attribution 4.0 International License (http://creativecommons.org/licenses/by/4.0/), which permits unrestricted use, distribution, and reproduction in any medium, provided you give appropriate credit to the original author(s) and the source, provide a link to the Creative Commons license, and indicate if changes were made. The Creative Commons Public Domain Dedication waiver (http://creativecommons.org/publicdomain/zero/1.0/) applies to the data made available in this article, unless otherwise stated. 


\section{Introduction}

Osteoarthritis (OA) is the most common form of arthritis worldwide [1]. Its symptoms are characterized by pain and reduced functional capacity, which make this disease the eleventh larger cause of years lived with disability [2].

The knee is the joint most commonly affected by OA, and it is responsible for significant morbidity and quality of life worsening [3]. About 6\% of 30-year-old adults or older have knee OA [4] and the prevalence of this disease increases by age. Estimated risk for its development is about $50 \%$ in individuals from the age of 85 years old [5].

OA treatment is based on pharmacological and non-pharmacological provisions [6]. Among medications used for fast pain relief, there are the analgesics, non-steroidal anti-inflammatory drugs and opioids. However, those medications do not often provide appropriate symptom improvement, and besides, they are associated to side effects, especially those gastrointestinal and cardiovascular ones [7]. Another therapeutic class used to manage OA is that of symptomatic slow-acting drugs, among which there are glucosamine sulfate and chondroitin sulfate. Nevertheless, the recommendations of use for such agents differ between international scientific organizations [8].

The variability in the quality of formulations $[9,10]$ and heterogeneity in the design and results of clinical trials with glucosamine sulfate and chondroitin sulfate are pointed out as factors for not recommending the use of those drugs in the current guidelines for OA treatment by the American College of Rheumatology (ACR) and Osteoarthritis Research Society International (OARSI) [11-13]. Differently, in Europe, the European Medicines Agency (EMA) approved the use of original glucosamine sulfate and chondroitin sulfate formulations, which follow the pharmaceutical quality standards, and the prescription is recommended as first line treatment for knee OA by Society for Clinical and Economic Aspects of Osteoporosis and Osteoarthritis (ESCEO) [14] algorithm. In Brazil, the general agreement for OA treatment by Sociedade Brasileira de Reumatologia states the use of glucosamine sulfate as a possibility for symptomatic OA treatment.

The objective of the present study was to evaluate efficacy and safety of combined glucosamine sulfate and chondroitin sulfate capsules (Eurofarma Laboratórios S.A.), for the treatment of knee OA, showing its non-inferiority for pain relief, versus combined glucosamine hydrochloride and chondroitin sulfate capsules (Cosamin DS ${ }^{\oplus}$, Nutramax Laboratories), whose efficacy has been demonstrated in previous clinical trials [15].

\section{Subjects and methods Study design}

Phase III, multicenter, randomized, double-blind, non-inferiority comparator, parallel group, active-controlled trial.
The trial was conducted from August, 2010 to March, 2011 in 4 Brazilian sites.

During the screening period, routine laboratory tests, knees X-ray, physical examination were performed, data was collected from clinical history and medications in use. Medications used for OA were discontinued and only paracetamol $750 \mathrm{mg}$ was allowed up to 4 times a day, in case of pain.

Subjects were randomized to one of the two study groups: fixed dose combined glucosamine sulfate $500 \mathrm{mg}$ and chondroitin sulfate $400 \mathrm{mg}$ (Eurofarma Laboratórios S.A.) or combination group of glucosamine hydrochloride $500 \mathrm{mg}$ and chondroitin sulfate $400 \mathrm{mg}$ (Cosamin DS ${ }^{\oplus}$ Nutramax Laboratories). In both groups, the medication was supplied as capsules, with the daily dose of 3 capsules.

Clinical visits were scheduled at 30 days (visit $2-$ V2), 60 days (visit 3 - V3), 90 days (visit 4 - V4) and 120 days (visit 5 - end-of-treatment visit) from the randomization visit (visit 1 - V1). Both treatments were administered throughout the trial, resulting in a maximum of 126 treatment days.

That trial was approved by the Research Ethics Committee from each participating study site. All procedures were performed in conformity with the Helsinki Statement and with resolutions no. 196, dated 10/10/1996 and no. 251, dated 08/07/1997, by Conselho Nacional da Saúde, following the Good Clinical Practices standards.

\section{Subjects}

Subjects over 40 years old, with a diagnosis of knee OA grades 1 to 3 as per Kellgren-Lawrence radiographic classification, clinical diagnosis of pain and functional limitation and score $\geq 4 \mathrm{~cm}$ in symptoms visual analog scale (VAS) (from 0 to $10 \mathrm{~cm}$ ) were enrolled. All subjects signed the informed consent form for the study. Exclusion criteria were: significant clinical history of joint trauma or surgery to be evaluated; presence of rheumatic disease responsible for secondary OA; severe joint swelling on physical examination; body mass index $(\mathrm{BMI})>30 \mathrm{~kg} / \mathrm{m}^{2}$; severe comorbidities likely to compromise the participation in the trial; use of systemic or intra-articular corticosteroids within the last 3 months; previous use of glucosamine and/or chondroitin; score $>$ 12 in Lequesne algofunctional index; joint arthroplasty to be evaluated; use of narcotic analgesic; pregnant, breastfeeding women or women with inappropriate use of contraceptives; any condition that, in the investigator's opinion, makes the subject unfit for the trial.

\section{Treatment and randomization}

The subjects eligible for the study were randomized at a 1:1 ratio for GS/CS test medication or Cosamin $\mathrm{DS}^{\oplus}$ comparator medication, at $\mathrm{V} 1$. The randomization process was performed independently, by means of a specific software, and a randomization list with four 
blocks was generated. This list was handed in an opaque, sealed envelope to the professional authorized to send the encoded medications to the research sites. That professional did not perform any other study procedure. Identical packaging was used to wrap the medication, with the purpose of blinding the treatment groups.

\section{Efficacy and safety evaluations}

The following efficacy evaluations were performed:

- Primary endpoint:

o Evaluation of analgesic efficacy by the investigator, performed at the end-of-treatment visit (V5), by means of a binary variant ("efficient" or "inefficient").

- Secondary endpoints:

o Overall efficacy evaluation, by means of a binary variant ("efficient" or "inefficient");

o Reduced pain intensity at V5 vs. V1, measured by visual analog scale (VAS) from 0 to $10 \mathrm{~cm}$, reported by the subject;

- Presence of joint pain by means of a binary variant ("pain" or "no pain");

○ Evaluation of joint pain by means of a four-point variant, with the following classifications: 1) no pain; 2) subject reports pain; 3) subject reports pain and changes facial expression; and 4) subject reports pain, changes facial expression and makes a move to cut the limb;

o Presence of joint swelling by means of a binary variant ("present" or "absent");

o Pain intensity measured by VAS $(0$ to $10 \mathrm{~cm})$ by the subject;

- Clinical efficacy as evaluated by the investigator at V5, based on pain VAS outcomes reported by the subject, with the following categories: 1) excellent; 2) very good; 3) good; 4) average; and 5) poor;

- Overall clinical efficacy as evaluated by the investigator at V5, based on pain parameters, rescue medication intake, clinical evaluation and responses by subjects in pain VAS, with the following categories: 1) excellent; 2) very good; 3) good; 4) average; and 5) poor;

- Use of rescue medication by means of a binary variant ("used" or "didn't use");

- Amount of rescue medication used (number of tablets taken).

Safety evaluations had as endpoints:

- Incidence of severe and non-severe adverse events, considered as potentially related to the study medication (possibly, likely or definitely related);
- Tolerability to study medication, with the following categories:

○ Excellent: subjects having no adverse events;

- Very good: subjects having 1 to 2 mild adverse events;

o Good: subjects having 1 moderate adverse event or 1 moderate adverse event and 1 mild one, or 1 moderate and 2 mild ones;

- Average: subjects having 1 moderate adverse event and 3 or more mild ones, or 2 moderate adverse events and 3 or more mild ones;

o Poor: subjects having 1 or more severe adverse event.

\section{Statistical analysis}

To calculate the sample size in outlining of non-inferiority proposed, it was considered that the test product primary endpoint, analgesic efficacy, would be at least not inferior to the comparator product. The non-inferiority margin considered was $10 \%$ with a type I $(\alpha), 0.05$ error and a type II $(\beta) 0.10$ error, and it was estimated that 100 evaluable subjects would be required [16, 17].

The populations analyzed for efficacy endpoints consisted of intention-to-treat (ITT) population, composed of all randomized subjects having an efficacy outcome at $\mathrm{V} 5$, and of per protocol (PP) population, composed of subjects from the ITT population attending all the visits provided in the protocol, and who had a minimum adherence of $75 \%$ to the study treatment. The safety population was composed of all subjects receiving at least one study treatment dose.

Primary efficacy analysis consisted of evaluation of the non-inferiority of the analgesic efficacy by the investigator, for GS/CS group, vs. Cosamin DS ${ }^{\bullet}$ group, and was performed in the per protocol (PP) population. That evaluation was also performed in the intention-to-treat (ITT) population, as a secondary analysis.

Concerning secondary analysis, a non-inferiority evaluation was also performed for reduced pain intensity, between V5 and V1. The other efficacy variants were analyzed by using the methodologies listed below.

An exploratory analysis was performed to investigate the relation between pain intensity decrease and efficacy evaluation by the investigator (based on pain VAS reported by subjects). It was performed with the purpose of studying how appropriate it would be to consider the treatments evaluated in categories "excellent", "very good", "good" and "average" as "efficient treatments".

The safety evaluation was performed by investigating the incidence of adverse events and tolerability to treatments.

Non-inferiority analyses used the two one-sided test (TOS) procedure, which builds $90 \%$ confidence intervals for the difference between treatments, in order to verify 
the test treatment non-inferiority vs. the reference treatment, with a 5\% significance level. Tests based on normal asymptotic distribution for the ratio difference and on Student $t$ distribution for the difference between means were used to calculate the associated descriptive level. For qualitative variants, tables with counting and percentage were made, 95\% confidence intervals were calculated for the ratios and q-square tests and Fisher Exact test to test the associations between variants.

For quantitative variants, tables with summary measures were built, 95\% confidence intervals were calculated for the means, Student t, Mann-Whitney tests and ANOVA and multiple Bonferroni comparisons were performed, to compare means.

Binomial test was used to study the subject condition evolution for qualitative variants.

\section{Results}

Demographic and baseline clinical characteristics

One hundred subjects were recruited for the trial. All of them met the eligibility criteria, and 50 subjects were assigned to test GS/CS group and 50 subjects to Cosamin $D^{\odot}$ group. Eleven subjects discontinued the study prematurely, 5 from GS/CS group and 6 from Cosamin $\mathrm{DS}^{\odot}$ group. Thus, 89 subjects were studied in the ITT population. Reasons and visits when study discontinuations occurred are described on Fig. 1. From the 89 randomized subjects, 3 subjects in the Cosamin $\mathrm{DS}^{\circ}$ group did not have minimum $75 \%$ adherence for the study total, and were excluded from PP population. Thus, the PP population consisted of 86 subjects (45 in the GS/CS group and 41 in the Cosamin DS ${ }^{\oplus}$ group).

Baseline characteristics of the population studied are shown on Table 1. Most subjects in the ITT population

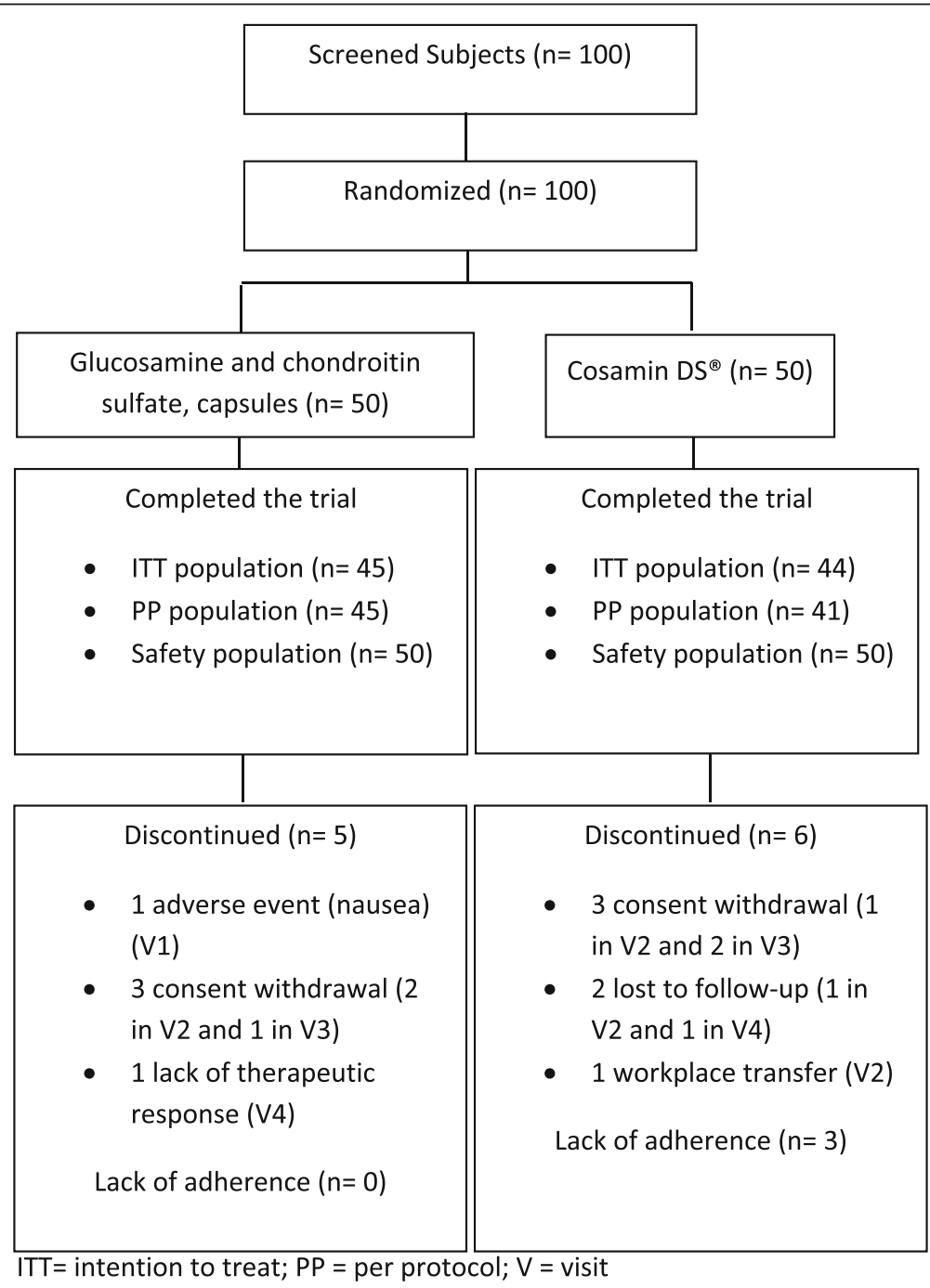

Fig. 1 Trial subjects flowchart. ITT intention to treat, PP per protocol, $V$ visit 
Table 1 Baseline clinical and demographic characteristics in ITT population $(N=89)$

\begin{tabular}{|c|c|c|c|}
\hline Characteristic & $\begin{array}{l}\text { Glucosamine sulfate } \\
\text { and chondroitin } \\
\text { sulfate group } \\
(n=45)\end{array}$ & $\begin{array}{l}\text { Cosamin } \\
\text { DS }{ }^{\circledR} \text { Group } \\
(n=44)\end{array}$ & $p$ value \\
\hline Age (years), mean $\pm S D$ & $63.4 \pm 8.3$ & $64 \pm 10.2$ & 0.753 \\
\hline Female, N (\%) & 43 (95.6) & $40(90.9)$ & 0.434 \\
\hline Color, white, N (\%) & $30(66.7)$ & $28(63.6)$ & 0.584 \\
\hline $\begin{array}{l}\text { OA diagnosis time } \\
\text { (years), mean } \pm S D\end{array}$ & $4.20( \pm 4.25)$ & $5.20( \pm 7.0)$ & 0.734 \\
\hline $\mathrm{BMI}\left(\mathrm{kg} / \mathrm{m}^{2}\right)$, mean $\pm \mathrm{SD}$ & $26.5 \pm 2.4$ & $26.7 \pm 2.9$ & 0.440 \\
\hline \multicolumn{4}{|c|}{ Kellgren and Lawrence classification, N (\%) } \\
\hline Grade I & $7(15.6)$ & $7(15.9)$ & 0.224 \\
\hline Grade II & $24(53.3)$ & $16(36.4)$ & \\
\hline Grade III & $14(31.1)$ & $21(47.7)$ & \\
\hline OA family history, N (\%) & $12(26.7)$ & $12(27.3)$ & 0.949 \\
\hline $\begin{array}{l}\text { Pain intensity, cm } \\
\text { (VAS; } 0-10 \mathrm{~cm} \text { ) }\end{array}$ & $6.72( \pm 1.61)$ & $6.36( \pm 1.45)$ & 0.313 \\
\hline \multicolumn{4}{|l|}{ Evaluation of joint pain, N (\%) } \\
\hline No pain & $1(2.2 \%)$ & $3(6.8 \%)$ & 0.361 \\
\hline Subject reports pain & $22(48.9 \%)$ & $19(43.2 \%)$ & 0.672 \\
\hline $\begin{array}{l}\text { Subject reports pain and } \\
\text { changes facial expression }\end{array}$ & $20(44.4 \%)$ & $20(45.5 \%)$ & $>0.999$ \\
\hline $\begin{array}{l}\text { Subject reports pain, } \\
\text { changes facial expression } \\
\text { and makes moves to } \\
\text { cut limb }\end{array}$ & $2(4.4 \%)$ & $2(4.5 \%)$ & $>0.999$ \\
\hline $\begin{array}{l}\text { Lequesne Index } \\
(0-24), \text { mean } \pm S D\end{array}$ & $8.57( \pm 2.2)$ & $8.58( \pm 2.47)$ & 0.931 \\
\hline \multicolumn{4}{|l|}{ Swelling, N (\%) } \\
\hline Absent & $37(82.2)$ & $36(81.8)$ & 0.960 \\
\hline Present & $8(17.8)$ & $8(18.2)$ & \\
\hline
\end{tabular}

$S D$ standard deviation, VAS visual analog scale, $B M I$ body mass index, ITT intention to treat, $O A$ osteoarthritis

were females, mean age of 64 years old, white and with a mean BMI of $26.5 \mathrm{~kg} / \mathrm{m}^{2}$, indicating overweight. About $27 \%$ of subjects reported OA family history. Kellgren and Lawrence radiographic classification grade II predominated. Mean Lequesne index was 8.5 and the mean pain intensity was $6.5 \mathrm{~cm}$ as per VAS. No significant differences were observed for clinical and demographic variants in the groups studied.

Mean adherence to the treatment was $93.7 \%$ in the GS/CS group and $93.1 \%$ on the Cosamin $\mathrm{DS}^{\circ}$ group, and there was no statistically significant difference between both groups ( $p=0.767)$.

\section{Efficacy results}

\section{Primary efficacy analysis}

The analgesic efficacy evaluated by the investigator in the GS/CS group was $88.9,95 \% \mathrm{CI}: 75.2-95.8 \%$ and in the Cosamin DS ${ }^{\bullet}$ group was $85.4 \%$; 95\%CI: 70.1, 93.4\% in the PP population. In the non-inferiority analysis, it was observed that the GS/CS group had a non-inferior result to that of the Cosamin $\mathrm{DS}^{\bullet}$ group, as shown on Fig. 2. Based on that analysis, efficacy in GS/CS group was 3.52\% higher than that of the Cosamin DS ${ }^{\bullet}$ group (90\%CI: - 8.39 to $15.43 \%, p=0.031$ ), and even disregarding the sampling error, that efficacy would be, in the worst case scenario, $8.39 \%$ lower to the efficacy in the Cosamin $\mathrm{DS}^{\circ}$ group, therefore, within the $10 \%$ non-inferiority margin that was set during the sample size calculations.

\section{Secondary efficacy analyses}

Tables 2 and 3 show the results for secondary efficacy endpoints. For all variants analyzed, no statistically significant differences were observed between the groups studied, both in the PP and in the ITT populations, after the 4 months of treatment.

Both groups had pain improvement. The rate of "no pain" subjects at the end-of-treatment visit was $66.7 \%$ (95\% CI: 50.9 to $79.6 \%$ ) in the GS/CS group and $52.3 \%$ (95\% CI: 36.9 to $67.3 \%$ ) in the Cosamin $\mathrm{DS}^{\circ}$ group, and the improvement was observed when compared to V1, statistically significant for both groups (ITT, $p<0.001$ ).

Mean reduced pain intensity as per VAS in the GS/CS group was $4.27 \mathrm{~cm}$ ( $95 \% \mathrm{CI}: 3.44$ to 5.12 ) and in the Cosamin DS ${ }^{\circ}$ group was $3.58 \mathrm{~cm}$ (95\% CI: 2.82 to 4.34), in the ITT population. Both decreases were statistically significant $(\mathrm{p}<0.001)$. GS/CS group was non-inferior to Cosamin $\mathrm{DS}^{\circ}$ group, with a mean difference of $0.692 \mathrm{~cm}$ in favor of the GS/CS group, with the non-inferiority margin magnitude used in that comparison of $0.358 \mathrm{~cm}$ (90\% CI: $-0.259 ; 1.642 ; p=0.035$ ). Figure 3 shows pain intensity evaluation by VAS at each study visit.

The evaluation of "treatment efficacy by the investigator", based on subject pain VAS was considered "excellent" and "very good" to about half the groups (57.9\% GS/ CS and 52.3\% Cosamin DS ${ }^{\circ}$. Results from variants "analgesic efficiency as per evaluated by the investigator" in the ITT population, "overall efficacy clinical evaluation by the investigator" with 5 categories, and its binary variant, "overall efficacy as evaluated by the investigator", also showed that the medication efficacy was observed by the investigator in both treatment groups.

The results of other secondary efficacy variants, including evaluations for swelling, joint pain evaluation with 4 categories, pain intensity as per VAS, use of rescue medication and amount of rescue medication intake, were also not statistically different from each other between the groups.

Exploratory analysis, evaluating the relation between pain intensity decrease and efficacy analysis by the investigator (based on subject pain VAS) showed that the "group" factor was not statistically significant for the 


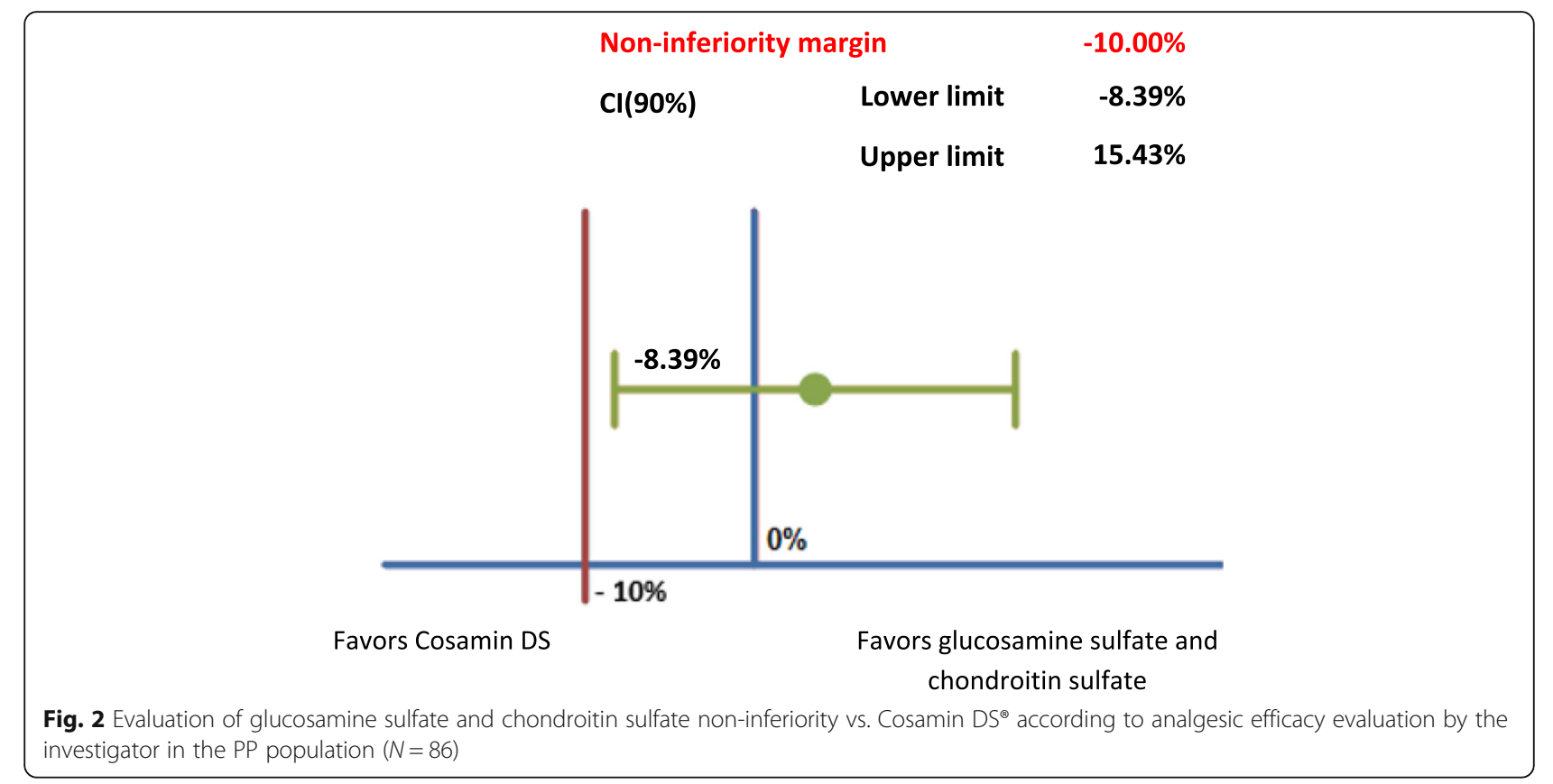

Table 2 Efficacy evaluations at the end-of-treatment visit in ITT population $(N=89)$

\begin{tabular}{|c|c|c|c|}
\hline & $\begin{array}{l}\text { Glucosamine sulfate } \\
\text { and chondroitin } \\
\text { sulfate }(N=45)\end{array}$ & $\begin{array}{l}\text { Cosamin } D^{\oplus} \\
(N=44)\end{array}$ & $p$ value \\
\hline \multicolumn{4}{|c|}{ Analgesic efficacy as evaluated by the Investigator, $N(\%)$} \\
\hline Efficient & $40(88.9)$ & $38(86.4)$ & 0.717 \\
\hline Inefficient & $5(11.1)$ & $6(13.6)$ & \\
\hline \multicolumn{4}{|c|}{ Overall efficacy as evaluated by the Investigator, $N(\%)$} \\
\hline Efficient & $34(75.6)$ & $35(79.5)$ & 0.652 \\
\hline Inefficient & $11(24.4)$ & $9(20.5)$ & \\
\hline $\begin{array}{l}\text { Reduced pain intensity } \\
\text { as evaluated by the } \\
\text { subject, VAS ( } 0 \text { to } \\
10 \mathrm{~cm} \text { ), average } \pm \mathrm{SD}^{\mathrm{a}}\end{array}$ & $4.27 \pm 2.83$ & $3.58 \pm 2.56$ & 0.230 \\
\hline \multicolumn{4}{|c|}{ Efficacy as evaluated by the investigator (based on subjects pain VAS), N (\%) } \\
\hline Excellent & $16(35.6)$ & $11(25.0)$ & 0.358 \\
\hline Very good & $10(22.3)$ & $12(27.3)$ & 0.630 \\
\hline Good & $6(13.3)$ & $6(13.6)$ & $>0.999$ \\
\hline Average & $8(17.8)$ & $9(20.5)$ & 0.793 \\
\hline Poor & $5(11.1)$ & $6(13.6)$ & 0.758 \\
\hline \multicolumn{4}{|c|}{ Clinical efficacy as evaluated by the investigator, $N(\%)$} \\
\hline Excellent & $8(17.8)$ & $8(18.2)$ & $>0.999$ \\
\hline Very good & $15(33.3)$ & $16(36.4)$ & 0.826 \\
\hline Good & $11(24.4)$ & $11(25.0)$ & $>0.999$ \\
\hline Average & $8(17.8)$ & $7(15.9)$ & $>0.999$ \\
\hline Poor & $3(6.7)$ & $2(4.5)$ & $>0.999$ \\
\hline
\end{tabular}

When compared to visit 1. SD standard deviation, VAS visual analog scale, ITT intention to treat relation $(p=0.504)$, and the "efficacy as evaluated by the investigator" factor (based on subject pain VAS) was statistically significant $(p<0.001)$. It was observed that the mean pain intensity decrease for subjects whose efficacy as evaluated by the investigator was classified as "ineffective" was statistically different from the means of groups of subjects to whom the treatment efficacy was considered "excellent", "very good", "good" and "average".

\section{Safety}

At least one adverse event was reported in 50 subjects, with 27 subjects (54.0\%; 95\% CI: 39.5 to $67.9 \%$ ) in the GS/CS group and 23 subjects (46.0\%; 95\% CI: 32.1 to $60.5 \%)$ in the Cosamin DS ${ }^{\odot}$ group. There was no statistically significant difference between groups $(p=0.549)$. The most commonly reported adverse events were flu and hyperglycemia, to which no relation with the treatment group was observed $(p=0.436$ and $p=0.674$, respectively). Only one subject, in the GS/CS group, discontinued the study due to an adverse event (nausea).

Thirteen subjects reported adverse events potentially related to the study treatment, with 8 (16\%; 95\% CI: 7.6 to $29.7 \%$ ) subjects in the GS/CS group and 5 (10\%; $95 \%$ CI: 3.7 to $22.6 \%$ ) subjects in the Cosamin DS ${ }^{\oplus}$ group, not statistically different from each other $(p=0.552)$. The most commonly reported adverse reactions corresponded to upper gastrointestinal tract (dyspepsia, epigastralgy, nausea and heartburn) and changes in glucose counts.

Four severe adverse events were reported, three from the Cosamin DS ${ }^{\oplus}$ group (fibromyalgia, left forearm fracture and right humerus fracture) and one in the GS/CS 
Table 3 Evaluation of joint pain and swelling and of the use of rescue therapy per visit in ITT population $(N=89)$

\begin{tabular}{|c|c|c|c|c|c|c|}
\hline & & \multicolumn{2}{|c|}{$\begin{array}{l}\text { Glucosamine sulfate and } \\
\text { chondroitin sulfate }(n=45)\end{array}$} & \multicolumn{3}{|c|}{ Cosamin DS ${ }^{\circledast}(n=44)$} \\
\hline & & $\mathrm{N}$ & $\%$ & $\mathrm{~N}$ & $\%$ & $p$-value \\
\hline \multirow[t]{5}{*}{ Absence of pain at joint pain evaluation } & V1 (Baseline) & 1 & 2,2 & 3 & 6,8 & 0.361 \\
\hline & V2 (Day 37) & 6 & 13,3 & 8 & 18,2 & 0.573 \\
\hline & V3 (Day 67) & 10 & 22,2 & 8 & 18,2 & 0.793 \\
\hline & V4 (Day 97) & 17 & 37,8 & 17 & 38,6 & $>0.999$ \\
\hline & V5 (Day 127) & 30 & 66,7 & 23 & 52,3 & 0.198 \\
\hline \multirow[t]{5}{*}{ Absence of joint swelling } & V1 (Baseline) & 37 & 82,2 & 36 & 81,8 & $>0.999$ \\
\hline & V2 (Day 37) & 37 & 82,2 & 37 & 84,1 & $>0.999$ \\
\hline & V3 (Day 67) & 41 & 91,1 & 36 & 81,8 & 0.230 \\
\hline & V4 (Day 97) & 42 & 93,3 & 39 & 88,6 & 0.485 \\
\hline & V5 (Day 127) & 44 & 97,8 & 42 & 95,5 & 0.616 \\
\hline \multicolumn{7}{|c|}{ Joint pain evaluation } \\
\hline \multirow[t]{5}{*}{ No pain } & V1 (Baseline) & 1 & 2,2 & 3 & 6,8 & 0.361 \\
\hline & V2 (Day 37) & 6 & 13,3 & 8 & 18,2 & 0.573 \\
\hline & V3 (Day 67) & 10 & 22,2 & 8 & 18,2 & 0.793 \\
\hline & V4 (Day 97) & 17 & 37,8 & 17 & 38,6 & $>0.999$ \\
\hline & V5 (Day 127) & 30 & 66,7 & 23 & 52,3 & 0.198 \\
\hline \multirow[t]{5}{*}{ Subject reports pain } & V1 (Baseline) & 22 & 48,9 & 19 & 43,2 & 0.672 \\
\hline & V2 (Day 37) & 28 & 62,3 & 25 & 56,8 & 0.669 \\
\hline & V3 (Day 67) & 22 & 48,9 & 27 & 61,4 & 0.289 \\
\hline & V4 (Day 97) & 20 & 44,4 & 20 & 45,5 & $>0.999$ \\
\hline & V5 (Day 127) & 9 & 20,0 & 25 & 34,1 & $<0.001$ \\
\hline \multirow{5}{*}{$\begin{array}{l}\text { Subject reports pain and changes } \\
\text { facial expression }\end{array}$} & V1 (Baseline) & 20 & 44,4 & 20 & 45,5 & $>0.999$ \\
\hline & V2 (Day 37) & 11 & 24,4 & 9 & 20,5 & 0.800 \\
\hline & V3 (Day 67) & 12 & 26,7 & 8 & 18,2 & 0.448 \\
\hline & V4 (Day 97) & 8 & 17,8 & 6 & 13,6 & 0.772 \\
\hline & V5 (Day 127) & 6 & 13,3 & 6 & 13,6 & $>0.999$ \\
\hline \multirow{5}{*}{$\begin{array}{l}\text { Subject reports pain, changes facial } \\
\text { expression and makes moves to cut limb }\end{array}$} & V1 (Baseline) & 2 & 4,4 & 2 & 4,5 & $>0.999$ \\
\hline & V2 (Day 37) & 0 & 0 & 2 & 4,5 & 0.242 \\
\hline & V3 (Day 67) & 1 & 2,2 & 1 & 2,3 & $>0.999$ \\
\hline & V4 (Day 97) & 0 & 0 & 1 & 2,3 & 0.494 \\
\hline & V5 (Day 127) & 0 & 0 & 0 & 0 & $>0.999$ \\
\hline \multirow[t]{4}{*}{ Subjects receiving rescue medication } & V2 (Day 37) & 39 & 86,7 & 40 & 90,9 & 0.739 \\
\hline & V3 (Day 67) & 41 & 91,1 & 36 & 81,8 & 0.230 \\
\hline & V4 (Day 97) & 41 & 91,1 & 38 & 86,4 & 0.522 \\
\hline & V5 (Day 127) & 36 & 80,0 & 33 & 75,0 & 0.619 \\
\hline \multirow{4}{*}{$\begin{array}{l}\text { Quantity of rescue medication } \\
\text { tablets taken (average } \pm \text { SD) }\end{array}$} & V2 (Day 37) & \multicolumn{2}{|l|}{$19,21 \pm 16,52$} & \multicolumn{2}{|c|}{$13,95 \pm 13,73$} & 0.109 \\
\hline & V3 (Day 67) & \multicolumn{2}{|l|}{$17,24 \pm 13,55$} & \multicolumn{2}{|c|}{$13,11 \pm 14,79$} & 0.148 \\
\hline & V4 (Day 97) & \multicolumn{2}{|l|}{$15,37 \pm 12,28$} & \multicolumn{2}{|c|}{$12,87 \pm 14,57$} & 0.351 \\
\hline & V5 (Day 127) & \multicolumn{2}{|l|}{$14,81 \pm 10,92$} & \multicolumn{2}{|c|}{$15,09 \pm 12,57$} & 0.920 \\
\hline
\end{tabular}

$S D$ standard deviation, $I T T$ intention to treat, $V$ visit 


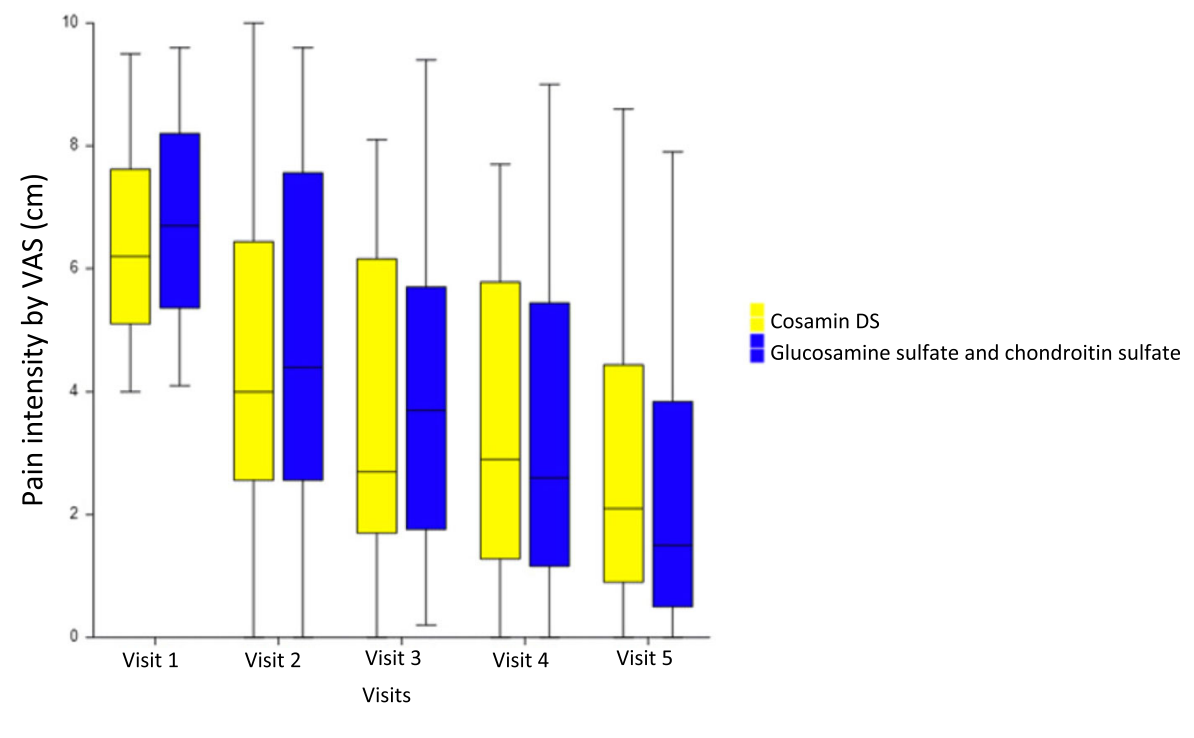

VAS, visual analog scale; ITT, intention to treat.

Fig. 3 Evaluation of pain intensity by VAS at each visit in ITT population $(N=89)$. VAS visual analog scale, ITT intention to treat

group (acute myocardium infarction). None was considered potentially related to the study treatment and the subjects were not discontinued from the study.

No deaths were reported in the course of the study.

\section{Tolerability evaluation}

Tolerability to the study treatments was evaluated as excellent or very good in 42 subjects (85.7\%) in the GS/CS group and in 41 subjects $(85.4 \%)$ in the Cosamin $\mathrm{DS}^{\circ}$ group. The evaluation was not performed to one subject in the GS/CS group and for 2 subjects in the Cosamin $\mathrm{DS}^{\circ}$ group. No statistically significant difference was observed between the groups studied $(p=0.967)$.

\section{Discussion}

This study showed the non-inferiority of the new fixed-dose combined formulation of GS/CS capsules, versus the combination already approved for use in the United States and Europe of glucosamine hydrochloride and chondroitin sulfate $\left(\right.$ Cosamin $\left.\mathrm{DS}^{\circ}\right)$ for $\mathrm{OA}$ pain relief.

The design used in this trial allowed to evaluate, in a comprehensive way, the new formulation efficacy, as both the investigator's point of view and the subject's were taken into account, within a randomized and double-blind scenario. Additionally, different tools were used to evaluate the trial endpoints, including, in addition to analgesic efficiency, overall efficacy evaluation, pain intensity evaluation and pain and joint swelling evaluation with physical examination. The choice of the comparator, Cosamin $\mathrm{DS}^{\star}$, was based on the registration of that product by EMA and by Food and Drug
Administration (FDA) for marketing in Europe and the United States, as at the time this trial was conducted, other products combined glucosamine and chondroitin were having their processes of registration adapted at Brazilian Health Regulatory Agency (ANVISA).

Efficacy results from clinical trials with combined glucosamine and chondroitin are conflicting in the literature. A systematic review with network meta-analysis did not find a clinically significant effect on pain improvement, with the use of that combination. On the other hand, a single trial directly comparing the combination vs. placebo was included in that analysis [18]. Another, more recent, network meta-analysis, showed that combined glucosamine and chondroitin have significant effects on pain relief and on functional improvement, when compared to placebo, and is also better than celecoxib in functional improvement [19]. The improvement of OA symptoms observed in the present trial verifies the efficacy of combined glucosamine and chondroitin.

Treatment efficacy evaluation by investigators considered as efficient for most subjects in this trial is supported by long-term results observed in randomized and real-life trials. In a randomized, double-blind, 6-month trial, in which combined chondroitin sulfate and glucosamine hydrochloride were compared to celecoxib in subjects with knee OA, with severe intensity pain, no differences were observed between the groups for pain improvement, joint stiffness, functional capacity and joint swelling evaluation, and the combined therapy was not inferior to the treatment with celocoxib [20]. In a long-term follow-up trial with subjects that had previously taken part in randomized and controlled trials, the 
treatment for at least 12 months with glucosamine sulfate delayed, significantly, the need for a knee arthroplasty for at least 5 years after treatment interruption [21]. Additionally, a reduction in the use of other medications for OA was shown, as well as in the number of medical appointment and tests related to that disease, on a long-term basis, with the use of that medication [19].

Safety profile and good tolerability to the treatment with combined glucosamine sulfate and chondroitin sulfate observed in this trial are consistent with literature data. In a meta-analysis comparing glucosamine and chondroitin safety, both as monotherapy or combined therapy to celecoxib and to placebo, it was observed that only celocoxib use was associated to a higher incidence of gastrointestinal adverse events, when compared to placebo [19].

The limitations to that study include the absence of a placebo group. As that study was conducted for the registry of the formulation studied, a design including a non-inferiority evaluation related to a medication with proven efficacy and approved for use by regulatory agencies was required. Another limitation is the small number of subjects enrolled in each arm of the trial, which was, however, based on literature [16, 17]. Additionally, no specific evaluations on physical function and subjects' quality of life were performed, as the trial focused on pain evaluation and overall treatment efficacy evaluation. The use of different formulations between the groups, glucosamine sulfate and glucosamine hydrochloride, may be pointed as another limitation for the trial, but once the formulas are dissociated in the stomach, glucosamine pharmacological effects seem not to difer [22].

\section{Conclusions}

The present study provides conclusive evidence that the new formulation of fixed dose combination of glucosamine sulfate and chondroitin sulfate capsules is efficient for the treatment of knee OA. Moreover, favorable safety profile and good tolerability make that formulation an option for OA treatment.

\section{Acknowledgements}

The authors thank the trial subjects and the team from each one of the sites involved in the clinical trial.

\section{Funding}

Eurofarma funded this study and participated in the study design; study research; collection, analysis and interpretation of data; and writing, review, and approval of this article. All authors had access to the data and participated in the development, review, and approval of this article and in the decision to submit it for publication.

\section{Availability of data and materials}

The data that supports the findings of this study are available on request from the corresponding author [A.B.V.L.]. The data are not publicly available due to the privacy of data from research participants.

\section{Authors' contributions}

$A B V L, J A M, G C B$ and MLC participated in the recruitment of subjects and in the conduction of the study. All authors read and approved the final manuscript.

\section{Ethics approval and consent to participate}

The study was conducted following international and local ethical standards and regulations, and was approved by the Independent Ethics Committee from each participating institution before its beginning, with the following sites and their respective RECs: Hospital Heliópolis Research Ethics

Committee (Centro Paulista de Investigações Clínicas Ltda.), Research Ethics Committee - UNICAMP (Instituto de Pesquisa Clínica e Assistência Medica de Campinas), Instituto do Câncer Dr. Arnaldo de Vieira de Carvalho Research Ethics Committee (Clínica Perdizes) and Faculdade de Medicina do ABC Research Ethics Committee (Instituto de Medicina Avançada).

\section{Consent for publication}

Not applicable.

\section{Competing interests}

Dr. Andrea B. V. Lomonte was a medical-scientific advisor and was paid by Eurofarma Laboratórios S.A. to write the scientific article. Dr. José Alexandre Mendonça and Dr. Gilberto C. Brandão were investigators in that study and were paid by Eurofarma Laboratórios S.A. to conduct the clinical trial. Dr. Marise L. Castro was paid by Eurofarma Laboratórios S.A. to conduct the trial and she participates in clinical trials sponsored by Laboratórios Aché, Sanofi, Eurofarma, Eli Lilly and Amgen.

\section{Publisher's Note}

Springer Nature remains neutral with regard to jurisdictional claims in published maps and institutional affiliations.

\section{Author details}

${ }^{1}$ Centro Paulista de Investigação Clínica (CEPIC), Rua Moreira e Costa, 342 Ipiranga, São Paulo, SP 04266-010, Brazil. " Instituto de Pesquisa Clínica e Assistência Médica de Campinas (IPECC), Campinas, SP, Brazil. ${ }^{3}$ Clínica Perdizes, São Paulo, S.P., Brazil. ${ }^{4}$ Instituto de Pesquisa Clínica e Medicina Avançada (IMA), São Paulo, S.P., Brazil.

Received: 27 April 2018 Accepted: 23 November 2018 Published online: 05 December 2018

\section{References}

1. Lawrence RC, Felson DT, Helmick CG, Arnold LM, Choi H, Deyo RA, Gabrie S, Hirsch R, Hochberg MC, Hunder GG, Jordan JM, Katz JN, Kremers HM, Wolfe F. Estimates of the prevalence of arthritis and other rheumatic conditions in the United States. Part II Arthritis Rheum. 2008:58(1):26-35.

2. Vos T, Flaxman AD, Naghavi M, Lozano R, Michaud C, Ezzati M, et al. Years lived with disability (YLDs) for 1160 sequelae of 289 diseases and injuries 1990-2010: a systematic analysis for the global burden of disease study 2010. Lancet. 2012;380(9859):2163-96.

3. Farr li J, Miller LE, Block JE. Quality of life in patients with knee osteoarthritis: a commentary on nonsurgical and surgical treatments. Open Orthop J. 2013;7:619-23.

4. Felson DT, Zhang Y. An update on the epidemiology of knee and hip osteoarthritis with a view to prevention. Arthritis Rheum. 1998;41(8):1343-55.

5. Murphy L, Schwartz TA, Helmick CG, Renner JB, Tudor G, Koch G, Dragomir A, Kalsbeek WD, Luta G, Jordan JM. Lifetime risk of symptomatic knee osteoarthritis. Arthritis Rheum. 2008:59(9):1207-13.

6. Coimbra IB, Pastor EH, Greve JMD, Puccinelli MLC, Fuller R, Cavalcanti FS, Maciel FMB, Honda E. Consenso brasileiro para o tratamento da osteoartrite (artrose). Rev Bras Reumatol. 2002;42(6):371-4.

7. Mosleh W, Farkouh ME. Balancing cardiovascular and gastrointestinal risks in patients with osteoarthritis receiving nonsteroidal anti-inflammatory drugs. Pol Arch Med Wewn. 2016;126(1-2):68-75.

8. Nelson AE, Allen KD, Golightly YM, Goode P, Jordan JM. A systematic review of recommendations and guidelines for the management of osteoarthritis: the chronic osteoarthritis management initiative of the U.S. bone and joint initiative. Semin Arthritis Rheum. 2014;43(6):701-12.

9. Volpi N. Quality of different chondroitin sulfate preparations in relation to their therapeutic activity. J Pharm Pharmacol. 2009;61(10):1271-80. 
10. Russell AS, Aghazadeh-Habashi A, Jamali F. Active ingredient consistency of commercially available glucosamine sulfate products. J Rheumatol. 2002; 29(11):2407-9.

11. Reginster JY, Cooper C, Hochberg M, Pelletier JP, Rizzoli R, Kanis J, Abadie E, Maheu E, Brandi ML, Devogelaer JP, Branco J, Herrero-Beaumont G, D'Hooghe $\mathrm{P}$, Bruyère $\mathrm{O}$. Comments on the discordant recommendations for the use of symptomatic slow-acting drugs in knee osteoarthritis. Curr Med Res Opin. 2015;31(5):1041-5.

12. Hochberg MC, Altman RD, April KT, Benkhalti M, Guyatt G, McGowan J, Towheed T, Welch V, Wells G, Tugwell P. American College of Rheumatology 2012 recommendations for the use of nonpharmacologic and pharmacologic therapies in osteoarthritis of the hand, hip and knee. Arthritis Care Res (Hoboken). 2012;64:465-74.

13. McAlindon TE, Bannuru RR, Sullivan MC, Arden NK, Berenbaum F, BiermaZeinstra SM, Hawker GA, Henrotin Y, Hunter DJ, Kawaguchi H, Kwoh K, Lohmander S, Rannou F, Roos EM, Underwood M. OARSI guidelines for the non-surgical management of knee osteoarthritis. Osteoarthr Cartil. 2014 22(3):363-88.

14. Bruyere O, Cooper C, Pelletier JP, Maheu E, Rannou F, Branco J, Luisa Brandi M, Kanis JA, Altman RD, Hochberg MC, Martel-Pelletier J, Reginster JY. A consensus statement on the European Society for Clinical and Economic Aspects of osteoporosis and osteoarthritis (ESCEO) algorithm for the management of knee osteoarthritis - from evidence-based medicine to the real-life setting. Semin Arthritis Rheum. 2016;45(4 Suppl):S3-11.

15. Das A Jr, Hammad TA. Efficacy of a combination of FCHG49 ${ }^{\text {TM }}$ glucosamine hydrochloride, TRH122 ${ }^{\text {TM }}$ low molecular weight sodium chondroitin sulfate and manganese ascorbate in the management of knee osteoarthritis. Osteoarthr Cartil. 2000;8(5):343-50.

16. Altman D. Practical statistics for medical research. Londres: Chapman \& Hall/ CRC; 1991. p. 73-7.

17. Kellner, S. R. O. Estatística sem mistérios. Volume I, segunda edição. Editora Vozes, p. 433-434.

18. Wandel $S$, Jüni $P$, Tendal $B$, Nüesch E, Villiger PM, Welton NJ, Reichenbach $S$, Trelle $S$. Effects of glucosamine, chondroitin, or placebo in patients with osteoartrhtis of hip or knee: network meta-analysis. BMJ. 2010:341:C4675.

19. Zeng C, Wei J, Li H, Wang YL, Xie DX, Yang T, Gao SG, Li YS, Luo W, Lei GH. Effectiveness and safety of glucosamine, chondroitin, the two in combination, or celecoxib in the treatment of osteoarthritis of knee. Sci Rep. 2015:5:16827.

20. Hochberg MC, Martel-Pelletier J, Monfort J, Möller I, Castillo JR, Arden N, Berenbaum F, Blanco FJ, Conaghan PG, Doménech G, Henrotin Y, Pap T, Richette P, Sawitzke A, du Souich P, Pelletier JP. Combined chondroitin sulfate and glucosamine for painful knee osteoarthritis: a multicentre, randomised, double-blind, non-inferiority trial versus celecoxib. Ann Rheum Dis. 2016;75(1):37-44.

21. Bruyere O, Pavelka K, Rovati LC, Gatterová J, Giacovelli G, Olejarová M, Deroisy R, Reginster JY. Total joint replacement after glucosamine sulfate treatment in knee osteoarthritis: results of a mean 8-year observation of patients from previous 3-year, randomised, placebo-controlles trials. Osteoarthr Cartil. 2008:16(2):254-60.

22. Block JA, Oegema TR, Sandy JD, Plaas A. The effects of oral glucosamine on joint health: is a change in research approach needed? Osteoarthr Cartil. 2010;18(1):5-11.

\section{Ready to submit your research? Choose BMC and benefit from:}

- fast, convenient online submission

- thorough peer review by experienced researchers in your field

- rapid publication on acceptance

- support for research data, including large and complex data types

- gold Open Access which fosters wider collaboration and increased citations

- maximum visibility for your research: over $100 \mathrm{M}$ website views per year

At $\mathrm{BMC}$, research is always in progress.

Learn more biomedcentral.com/submissions 